\title{
Corrigendum
}

\section{De novo chronic graft-versus-host disease presenting as hemolytic anemia following partially mismatched related donor bone marrow transplant}

\author{
K Godder, AR Pati, SH Abhyankar, LS Lamb, W Armstrong and PJ Henslee-Downey
}

Bone Marrow Transplantation 1997; 19: 813-817

In the third paragraph of the Results section, the dose for pulse high-dose steroids was given incorrectly as $500-1000$ $\mathrm{mg} / \mathrm{kg}$ instead of $500-1000 \mathrm{mg} / \mathrm{m}^{2}$, however the correct dose was given in Table 3 . The corrected version of this paragraph follows.

All five patients had been maintained on GVHD prophylaxis with cyclosporine; however, four were taken off steroid therapy within 2 weeks prior to presentation. Treatment of HA was initiated with pulse high-dose steroids (500$1000 \mathrm{mg} / \mathrm{m}^{2} \times 2$ doses) and daily intravenous gammaglobulin $\times 3-5$ doses and continued as shown in Table 3 . Response was observed in $4 / 5$ patients within 1 week and complete recovery that allowed tapering of therapy within 4 weeks. However, one of these patients (DH) required retreatment for a transient recurrence of HA. In the remaining patient (HR), delay in diagnosis and treatment was associated with a slow reponse ( 3 weeks) and the need for continuous high-dose intensive immunosuppressive therapy using four drugs (pulse high-dose steroids, IVIG, cyclosporine and azathioprine). Three patients continued to manifest limited skin cGVHD symptoms once immunosuppressants were tapered and had to be maintained on cyclosporine alternating with low-dose steroids. ${ }^{19}$ 